\title{
Message Indexing Based Car-theft Prevention Through Smartphone
}

\author{
Vishal Sharma ${ }^{1}$, Abhishek Gulati ${ }^{2}$, Manvi Madan ${ }^{3,}$ Kajal Puri $^{4}$ \\ ${ }^{1}$ (Assistant Professor, Dept of CSE, Bharati Vidyapeeth College of Engineering Delhi, India) \\ ${ }^{2,3,4}$ (Scholars, Dept of CSE, Bharati Vidyapeeth College of Engineering Delhi, India)
}

\begin{abstract}
Nowadays cars are amongst the most valuable asset for people, hence their security is a major concern for both the people as well as the car manufacturing companies. With the growth in the VLSI Technology has empowered the smart phones of this era to accommodate a solution to everyday problems of the users. Therefore, a system has been proposed which uses the resources of smart phone to prevent car-theft. The system has been developed primarily for Android Operating system and it successfully notifies the user within fraction of seconds in case of a theft-attempt by an unauthorised user of the car. The system empowers the users to ensure the safety of their cars through their smart phones.
\end{abstract}

\section{Keywords -Android Studio, Arduino, GSM, IR Sensor, Smartphone}

\section{INTRODUCTION}

In the past few decades, a new domain of research has emerged namely Internet of Things which provides motivation to bring together the hardware technologies with the software's developed and enhance the quality of living for the people across the globe. The vision to tie the whole world in a strand of connectivity seems achievable with the robust systems that include life changing applications. With the developments in VLSI technology, the capabilities of the smart phones have multiplied manifolds. Also, the smart phones are now capable of providing resources efficiently for applications that deals with heavy data [1] [2] [3]. Therefore, researchers are keen about exploring the capabilities of these smart devices. Since cars are a very important asset to people and people invest hefty portions of their income into it, their security is of prime importance. Moreover, the companies have to pay humungous amounts in case of car theft of insured cars to their customers therefore the companies are equally worried about the safety of the cars.

The system is yet another exploration in the domain of mobility by providing an efficient and highly useful application for general people. The application has been designed to alert the user in case of an unauthorized access to the user's car on their Smartphone device. In the era of digital technologies, an application thatan prevent the robbery of cars can be a great boon for car owners. The application has been designed, deployed and tested for Android Operating System. The major reason for the selection of Android OS is its widespread popularity. The other reasons that have contributed to the adoption of android operating system are impeccable user interface, open source and hardware independent development platform. Also, android provides a platform to develop applications for automobiles via it Android Auto development feature [4]. The application has been designed for the use of general people but can be optimized for commercial use as well to ensure the theft prevention of cars. Further, the application has great utility in everyday scenario especially for people staying in areas where there is no human based security available for cars. The application can easily eliminate the need to appoint security guards and thus helps eliminate unnecessary cost involved in securing the cars. Hence, it can be safely concluded that the application has wide usage in today's scenario.

The prime objective of the paper is to present a system that has been developed to prevent car-theft. The android application has been developed that determines the threat to the security of the car by extracting the text from the text messages received by the android phone. The text retrieved by the application is classified using an approach inspired from the technique presented in [5].

The system has been developed with intent to enhance security of the cars and eliminate the need for the humans to keep a check at the cars by being physically present near them.

\section{LITERATURE SURVEY}

A number of existing works in the domain of mobility and security system has been studied before and during the designing of the system. A literature survey of the existing works has been presented below:

In [5], the authors have presented a prototype utilizing the capabilities of GSM module to connect the user and the monitoring system. The monitoring is done through the embedded systems that detect the changes in the 
environmental conditions. The changes sensed by the embedded system generate the activation signal for the GSM module. The GSM module sends a Short Message Service (SMS) to the user's mobile number. Here, SMS is being used to alert the user about the changes observed by the embedded system. The prototype worked satisfactorily and is cost efficient. Hence this system can be used with dedicated circuits for real-time critical operations. This prototype has been used in the system for automatic surveillance of cars to prevent theft.

In [6], the authors have proposed a system where the air pollution caused by the cars has been automatically monitored using an embedded system. The system detects the breach of air pollution threshold levels and generates a buzz. This buzz is used as an activation signal for the rest of the challan generation circuitry. If the air pollution caused by the car remains above the threshold levels for 3 days that is if the buzz remains for 3 consecutive days, then a challan is generated for the user. However, the system does not incorporate any module to indicate the user or the police regarding the high air pollution levels. The system has an auto-monitoring feature along with an android application to bridge the communication between the user and the embedded system deployed at the car.

In [7], the authors proposed a system for monitoring kitchen parameters in real-time automatically. The parameters such as room temperature, light intensity, fire detection, motion detection and LPG gas levels are being auto-monitored in the system. Whenever abnormal conditions are sensed by the system, the status of kitchen is reported to the user on their mobile phone through GSM module. The user can then take appropriate action by using any AT commands or by taking necessary steps through their e-mail. The e-mail id is password protected and can be used to send required steps to be taken at the kitchen through dedicated web pages. The test results of the prototype were promising.

In [8], the authors present an anti-theft tracking system for car through the use of only GSM module. The system can be turned ON and OFF through a Short Message Service (SMS) and will report the location of the car through SMS as well. The location is determined by tracking the time gap between the two messages and the last location in reference to the nearest Base Station. The most significant drawback of the system is that it does not ensure whether or not a theft attempt has been made. The system sense the RFID card number to authenticate the authorized user and in case that security is breached, the system ensures if there is anybody sitting in the car or not through the IR sensor deployed in the car. Also, the GSM module continues to stay OFF till the user sends an ON message to the GSM module at the car in [8]. This is the most significant drawback of the system as the theft attempt can be made during the time span when the user has not switched the module ON which can happen due to a lot of reasons. Moreover, the system is synced to an android application which continuously checks the text of all the messages retrieved at the phone and when it is an alert message from the theft monitoring system, the application automatically generates a sound notification.

In [9], the authors have proposed a system that is an integration of a GSM and GPRS modules with a microcontroller unit. The hardware module has a companion smart phone application which with the help of Google Maps API represents the location of the vehicle to be tracked.

In [10], the authors have proposed a new methodology of localization of a smartphone by the use of existing GPS, Wi-fi and GSM technology in the smartphone. The paper also gives a detailed literature survey of the existing technology the is being used in smartphone for location and tracking. The new method being proposed keeps a track of user activities on his mobile phone to ensure exact location tracking.

\section{PROPOSED SYSTEM}

The system includes various phases for processing the information necessary to determine an unauthorized access to the car. These phases collectively contribute to a system response which notify the user in case of a theft-attempt. These various processes have been depicted in the Block Diagram presented in the Fig. 1 below.

The various phases and the functionality performed at them have been elucidated below:

\subsection{Text Message Generation}

The system is initiated when a signal is generated by the circuitry installed at the car. The main purpose of the circuitry is to identify access attempt made by an unauthorized user of the car.

This is determined by checking whether someone has entered the car carrying an authorized RFID Card or not. The IR Sensor senses if anyone has entered the car. The authenticated RFID Card is recognized through the unique authenticated string generated by the RFID Card. If someone enters the car without showing the authenticated RFID card, the GSM module is activated.GSM is an SMS activation system which detects abnormal behaviour and communicates to a remote telecare control centre, the clients, and their caregivers 
[7].The signal generated at the GSM module invokes a text message sent to the pre-defined phone number indicating the danger to the car. The text message generated has a predefined text indicating the theft-attempt made on the car.

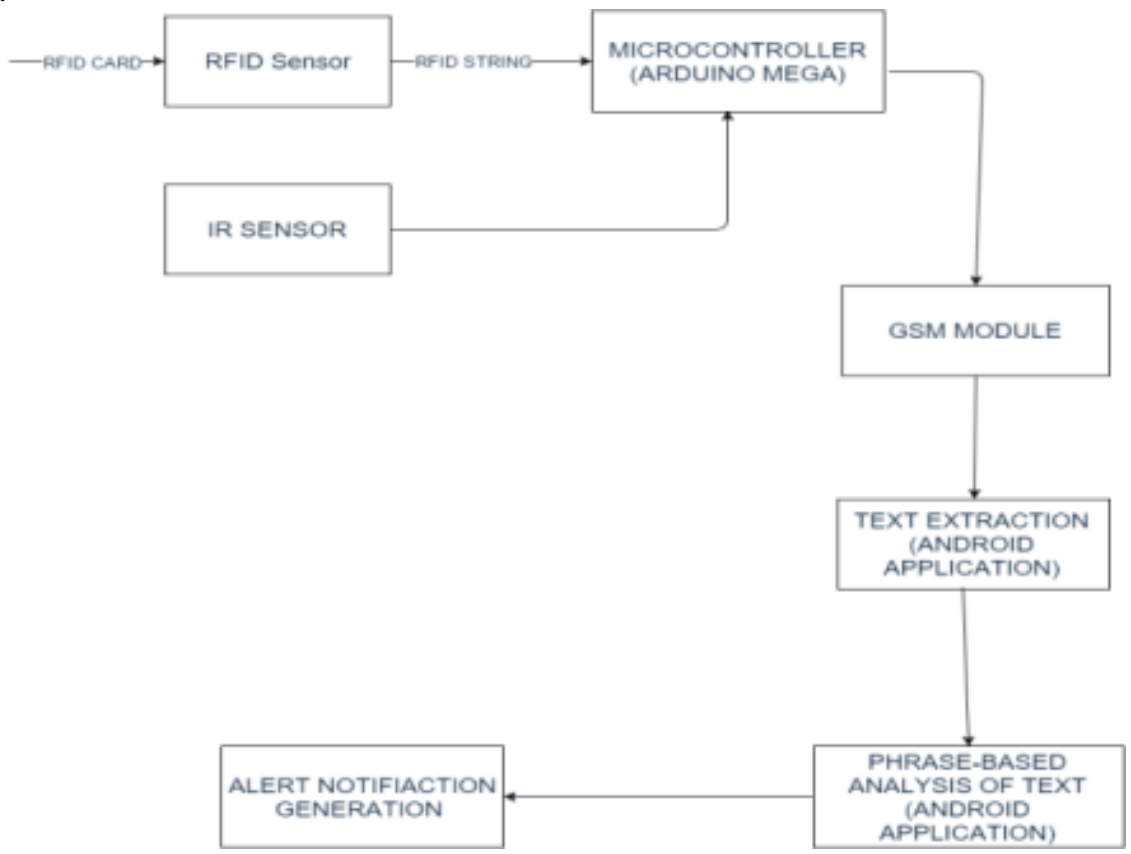

Fig. 1: Block Diagram of proposed system

\subsection{Text Message Received}

The text message generated by the circuitry at the car is received by the application deployed at the android device. The android application is deployed at the car owner's smartphone to ensure that the message reaches the car owner at the earliest.

\subsection{Text Extraction}

Text retrieval from the entire text message is done by the Text Extraction module of the application. The module retrieves all the text from the text message from the inbox of the android phone's message box. All the text retrieved is sent to the further phases to acknowledge whether or not the text message indicating the theftattempt has been received.

\subsection{Classification of the Text}

The classification of the text is done using an approach that is similar to the approach used in[5]. The message text extracted in the previous phase is broken down into sets of phases and words. The sets of phrases and words that indicate the theft-attempt are classified beforehand during testing phase. These training sets of phrases and words are determined by the kind of text message being generated by the circuitry deployed at the car. These classified sets of phrases and words identified during training and sets of words and phrases extracted by the text messages in the inbox are sent to the next phases to process further.

\subsection{Searching for Appropriate Phrases/ Words in Retrieved Text}

The extracted sets of phrases and words are then matched with the predetermined sets of phrases and words that were obtained while determining training sets. While searching the text messages extracted from the inbox, if a match is found. The flag is set true and sent further to indicate the same.

3.6 Notification Generation

If the flag in the previous phase is set to true, then a notification has to be generated to alert the user regarding the theft attempt being made by an unauthorized user of the car. Otherwise, no notification is to be generated.

The notification sound is predetermined by the user while setting up the application. The user can set the sound based on what sound will he be able to distinctly recognise in case of an emergency. The sound notification will continue to alert the user till the time the user ensures the application that he is aware of the security status of the car. 


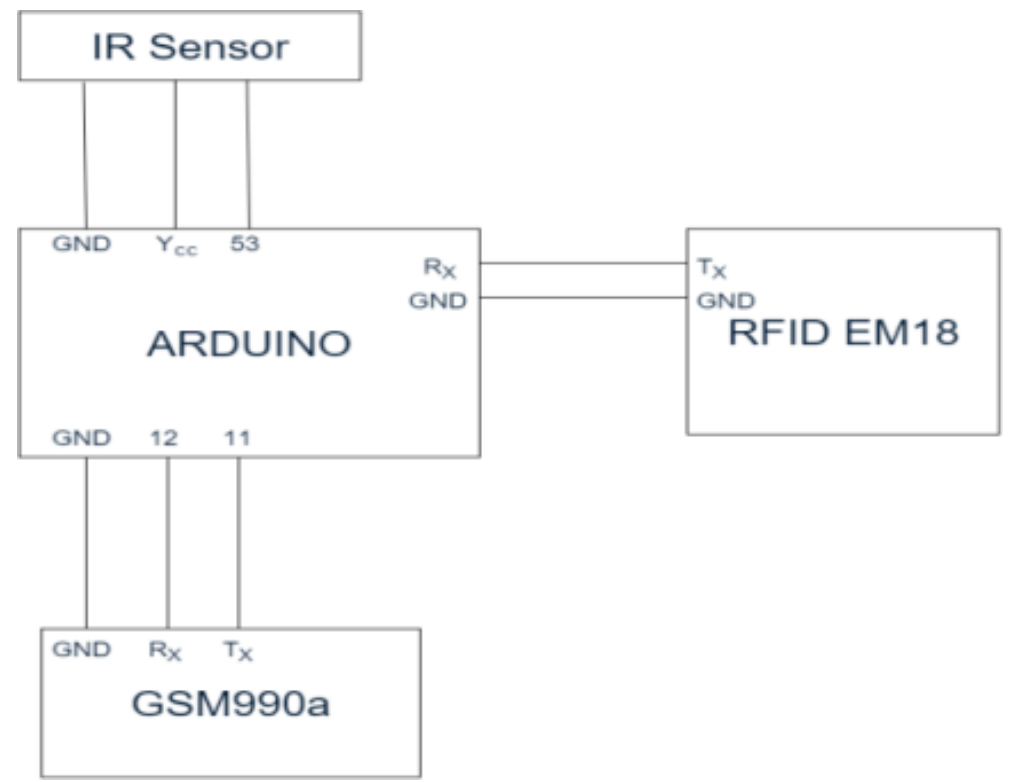

Fig. 2: Blackbox Circuit Diagram of the embedded system deployed at the car

In Fig. 2, the blackbox circuit diagram for the embedded circuitry has been shown. The system consists of an IR sensor, a RFID sensor, an Arduino and a GSM module. The system starts with a RFID sensor connected to the Arduino through transmission pin and ground pin. The Arduino receives the signals generated from the RFID sensors. The RFID sensor gives out signals using an RFID card. The Arduino is further connected to an IR sensor which transmits signal if any object comes in front of the sensor. The voltage is provided by the $Y_{c c}$ Pin in Arduino. These signals are processed by the Arduino and according to the algorithm sends out signals to the GSM module connected directly to the Arduino through transmission pin (Tx), Receive pin (Rx), and a ground pin.

The GSM module is powered by an external power show which can be any regular power source.Arduino processes the signals received from the IR sensor and RFID sensor. The algorithm installed in the Arduino checks whether the signals are legitimate or not. If signals indicate the illegitimacy of the IR sensor reading or the RFID sensor reading then it sends out a signal to the GSM module which commands it to send out the ALERT message to the registered mobile Number. Hence, the Alert is generated on illegal access to the system.

\section{SOFTWARE AND HARDWARE REQUIREMENTS}

The software and hardware specification for the system to be developed has been described below:

\subsection{Software Requirements}

The most efficient and reliable SDK available for the development of android applications is Android Studio IDE [11]. Since Android Studio [11] is the official development kit for android applications, it is equipped with the most advanced development tools from Google. The development kit is based on IntelliJ[12]IDEA and provides a flexible development environment because of the Gradle[13]-based built system. The GUI development has rich options through drag and drop facility of pre-defined widgets. Customized widgets can also be created to enhance GUI. Android Studio has emulators for various API versions of Android for testing purposes.

Genymotion [14] Android emulator has been used during the development of the application for testing the app for various hardware configurations. Genymotion is an Android emulator for building and testing great Android apps. It's fast, simple and powerful.

\subsection{Hardware Requirements}

As the product is a theft prevention application that needs hardware and software modules, its hardware requirements are nontrivial. For reasonable performance a dedicated web server and database server are required.

Following requirements are for client side for running the website on their desktop. To implement this software part in the system following things are required: 
4.2.1 ARDUINO Mega -The microcontroller which stores and processes the embedded code.

4.2.2 GSM 900A - The GSM module that is used to emulate a mobile phone. It is responsible for sending the 4.2.3 Short Service Message (SMS) to the user from the embedded circuitry at the car.

4.2.4 RFID card - The card that is used to authenticate the authorized user from the unauthorized one. In other words, it is the key to enter the car.

4.2.5 IR sensor - The sensor that determines if anybody has entered the car or not by using the Infrared rays.

4.2.6 Connecting wires - The wires that are used to assemble components of the embedded system.

4.2.7 SIM card - For the GSM module with Short Service Message (SMS) service activated.

\section{INTERFACES AND DISCUSSION}

The various interfaces of the application have been shown below in Fig. 3, 4, 5, 6. The functionality expressed in the given interfaces can be explained as below. In Fig. 3, the predetermined term has been identified to be present in the set of phrases/ words extracted from the text message and therefore the notification has been generated. In Fig. 4, the text message does not contain the phrase/ word in the received text message, hence no threat has been detected. Also, no sound notification has been generated. In Fig. 5 and 6, the interface of the application's message inbox has been shown.

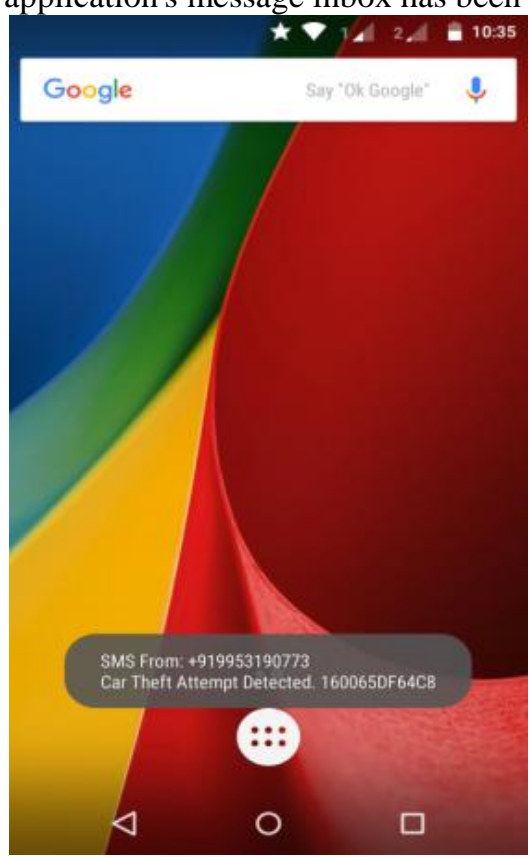

Fig. 3: Interface of the application when threat detected

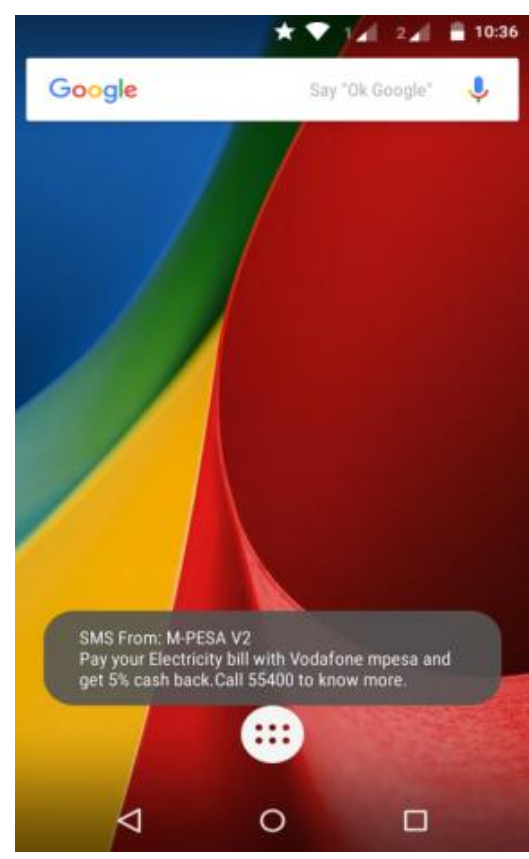

Fig. 4: Interface of the application when no threat has been detected 


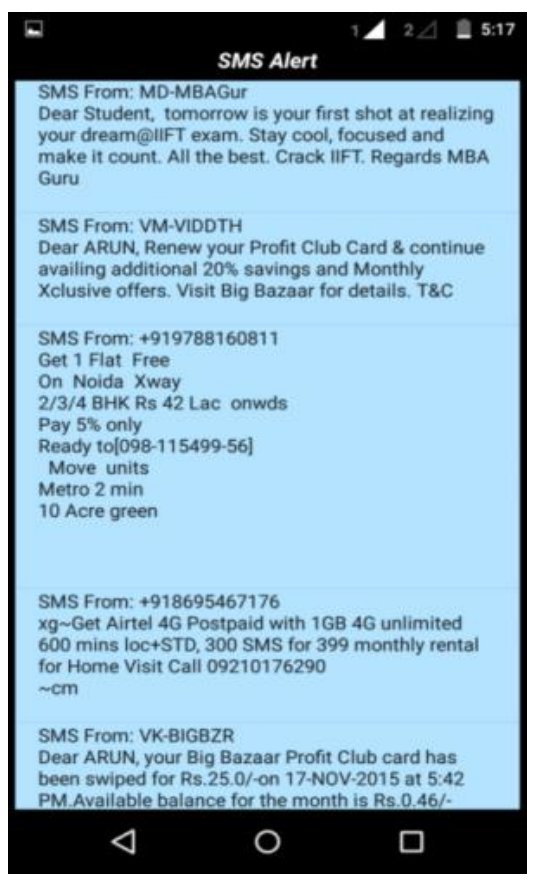

Fig. 5: Interface of the application when no message has been recently received

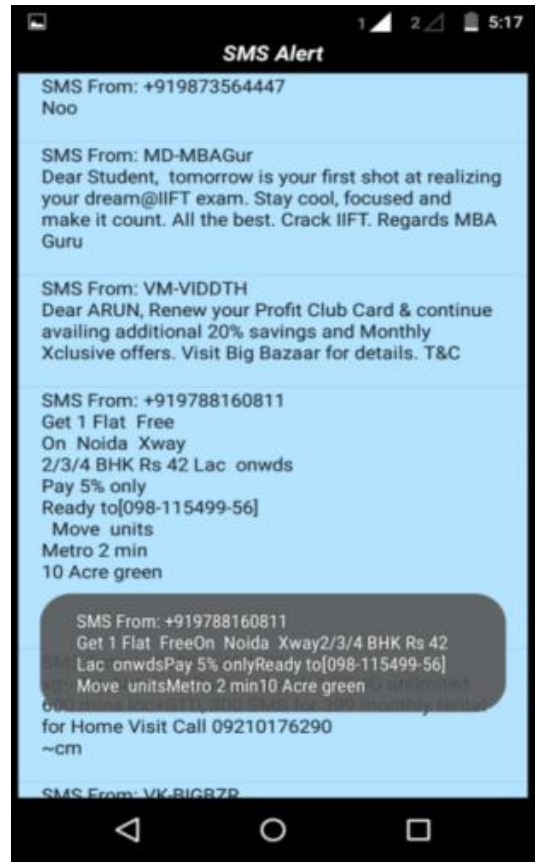

Fig. 6: Interface of application inbox while particular message been received

\section{CONCLUSION}

The system has been tested in real-time to ensure its efficiency and it is successfully notifying the user in case of an unauthorized access to the car. Therefore, it can be concluded that the system is robust, cost-efficient and the Android application used to provide the interface to the system is easy to use. In future, the aim is to enhance the functionality of the system by integrating other important assets of the general use and preventing their robbery or prevent unauthenticated access to them.

\section{REFERENCES}

[1] Google maps. (2015, December). Retrieved from https://maps.google.com/

[2] Whatsapp.(2015 December). Retrieved from https://www.whatsapp.com/.

[3] Google Play Store. (2015, December). Retrieved from https://play.google.com/store/apps/details?id=com.truecaller\&hl=en.

[4] Android Developers Automobile. (2015, December). Retrieved from http://developer.android.com/auto/index.html.

[5] Thangarajah, Akilan, BuddhapalaWongkaew, and MongkolEkpanyapong, Implementation of Auto Monitoring and ShortMessage-Service System via GSM Modem, IJCER 3.2, 2014, 63-68.

[6] Chandrasekaran, Siva Shankar, ShivkumarMuthukumar, and SabeshkumarRajendran, Automated control system for air pollution detection in vehicles, Proc. Intelligent Systems Modelling \& Simulation (ISMS), 2013 4th International Conference on.IEEE, 2013, 49-51.

[7] Sahani, Mrutyunjaya, Avinash Nayak, Rishabh Agrawal, and Debadutta Sahu, A GSM, WSN and embedded web server architecture for Internet based kitchen monitoring system, Proc. Circuit, Power and Computing Technologies (ICCPCT), International Conference on.IEEE, 2015,1-6.

[8] Swapna, Pagadala, and Syed Jilani Pasha, Anti-Theft Tracking System for Automobiles, International Journal of Scientific Engineering and Technology Research, 4 (25),2015,4875-4878.

[9] Lee, SeokJu, Girma Tewolde, and Jaerock Kwon, Design and implementation of vehicle tracking system using GPS/GSM/GPRS technology and smartphone application, Internet of Things (WF-IoT), World Forum on. IEEE, 2014.

[10] Papliatseyeu, Andrei, and Oscar Mayora, Mobile habits: Inferring and predicting user activities with a location-aware smartphone, Proc.3rd Symposium of Ubiquitous Computing and Ambient Intelligence 2008. Springer Berlin Heidelberg, 2009.

[11] Android Developers, (2015, December) Retrieved from http://developer.android.com/index.html.

[12] JetBrains, (2015, December) Retrieved from https://www.jetbrains.com/idea/download/\#section=linux/.

[13] Gradle, (2015, December). Retrieved from http://gradle.org/the-new-gradle-android-build-system/.

[14] Genymotion, (2015, December). Retrieved from https://www.genymotion.com/\#!/. 